\title{
A Case of Primary Subpleural Pulmonary Microcystic Myxoma Coincidentally Occurred with Pulmonary Adenocarcinoma
}

\author{
Jungsuk Ahn · Na Rae Kim • Seung Yeon Ha • Keun-Woo Kim ${ }^{1}$ Kook Yang Park ${ }^{1}$ Yon Mi Sung ${ }^{2}$ \\ Departments of Pathology, ${ }^{1}$ Thoracic Surgery, and ${ }^{2}$ Radiology, Gachon University Gil Medical Center, Incheon, Korea
}

Myxoma occurs most commonly in the heart, which is regarded as the origin of primitive mesenchymal cells that differentiate into multiple tissues. Up to $18 \%$ of cardiac myxomas are located in the right atrium and are frequently diagnosed after recurrent pulmonary embolism. ${ }^{1}$ Extracardiac myxomas have been reported in the subcutaneous tissue, muscle, and lung. ${ }^{2,3}$ Nine cases of primary pulmonary myxoma have been described. ${ }^{4,5}$ Due to its rarity, clinical and histologic review of pulmonary myxoma is limited in number.

To the best of our knowledge, this is the first report describing cytologic features of a primary pulmonary microcystic myxoma.

\section{CASE REPORT}

A 71-year-old Korean man was admitted for an incidentally detected pulmonary mass. The initial chest X-ray and subsequent computed tomography (CT) showed a subpleurally located, well-defined, round and smooth mass in the left upper lobe, measuring $1.0 \times 0.8 \times 0.7 \mathrm{~cm}$ in size (Fig. 1A). The surrounding parenchyma showed mild volume loss and focal consolidation, measuring about $2.0 \times 2.0 \times 1.8 \mathrm{~cm}$. A poorly enhanced soft tissue lesion, measuring $3.5 \times 3.0 \times 2.0 \mathrm{~cm}$ in size, was also found in the anterior thymic area. Cardiac echographic findings were as follows; normal-sized cardiac chambers, normal left ventricular systolic function, normal left ventricle filling pattern, and no

\footnotetext{
Corresponding Author

Na Rae Kim, M.D.

Department of Pathology, Gachon University Gil Medical Center, 21 Namdong-daero 774 beon-gil, Namdong-gu, Incheon 405-760, Korea

Tel: +82-32-460-3847, Fax: +82-32-460-2394, E-mail: clara_nrk@gilhospital.com
}

Received: February 7, 2015 Revised: March 10, 2015

Accepted: March 12, 2015 motional abnormalities or regional wall abnormalities. Under the impression of pulmonary hamartoma, a wedge resection of the subpleural mass and partial thymectomy through video-assisted thoracoscopic surgery was performed. A 3-month follow up was planned for the parenchymal consolidation.

Grossly, the wedge-resected lung showed a well-delineated, glistening, round, and gray subpleural mass (Fig. 1B). The cut surface of the mass was gelatinous mucoid, grayish yellow without a capsule. Intraoperative touch imprints were made using Papanicolaou and hematoxylin and eosin stains. The smears were paucicellular showing copious amounts of viscous pinkish mucoid material and scattered inflammatory cells (Fig. 2A). Scattered inflammatory cells such as macrophages containing foamy granular cytoplasm with occasional hemosiderin-laden macrophages were found. Cracking and a pleated pattern were observed on thickly smeared slides. Sparse capillaries were also found. There were a few loose aggregates of bland, monomorphic spindle cells with oval nuclei and no distinct nucleoli, and these spindle cells had long, thin, and tapered cytoplasmic processes (Fig. 2B). The nuclei were round to oval with indistinct nucleoli, and the cytoplasm was vacuolated and granular with a well-delineated cell border (Fig. 2C). Differential diagnoses for the frozen section were myxoid tumors including pulmonary hamartoma.

The specimen was fixed with $10 \%$ formalin, and paraffin-embedded tissue was stained with hematoxylin and eosin. Microscopically, the pulmonary mass was composed of scattered spindle-shaped mesenchymal cells with chronic inflammatory cells, embedded in a rich basophilic gelatinous myxoid matrix (Fig. 2D). Stellate cells with vacuolated bubbly cytoplasm were observed. Neither mitosis nor cytologic atypia was found in the stellate cells. In the abundant basophilic mucoid matrix, there were no cellular elements other than chronic inflammatory cells 

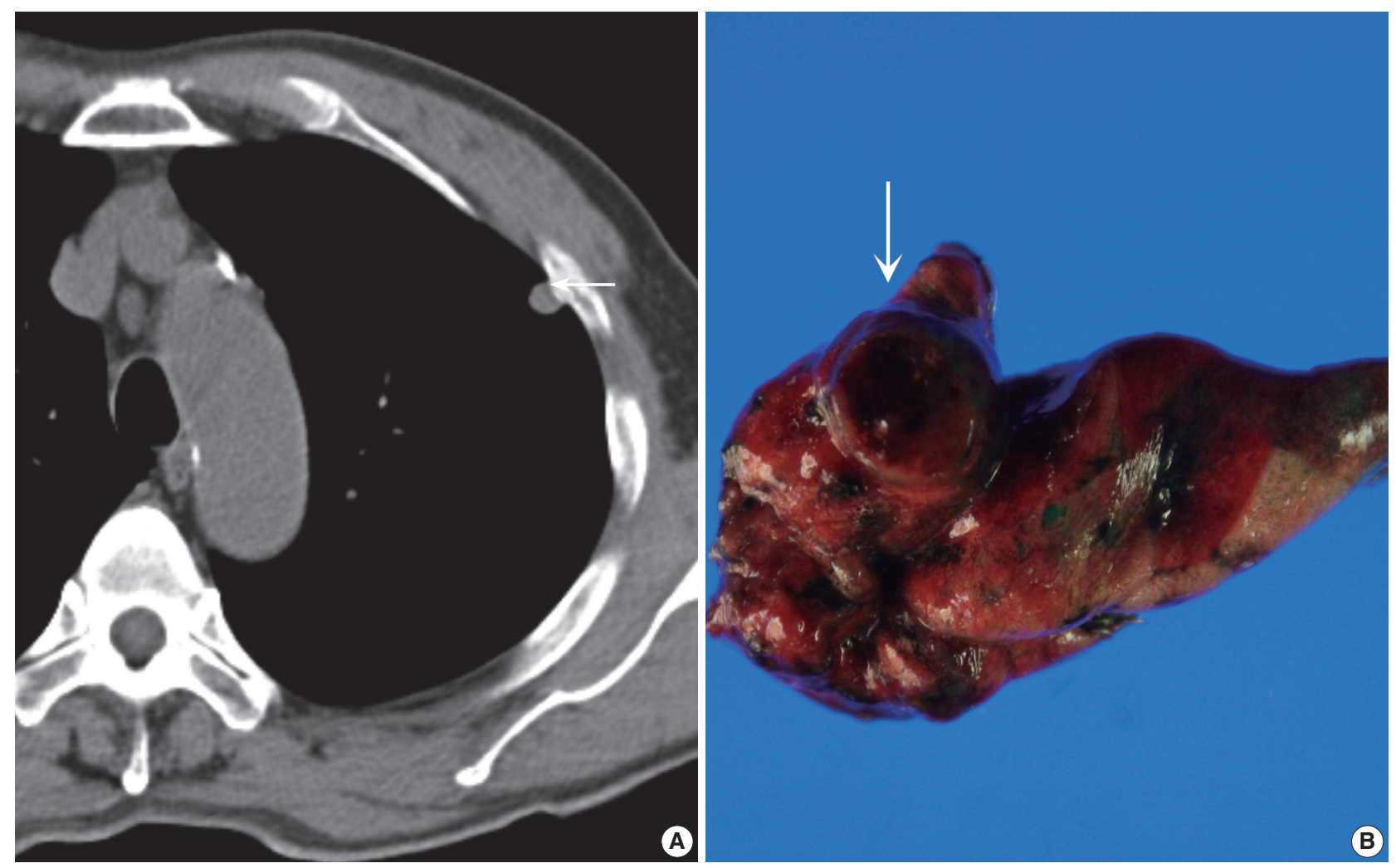

Fig. 1. (A) Precontrast chest computed tomography shows a well-delineated low-attenuated oval mass (arrow) with slightly high density delete the portion in the subpleural area. (B) Gross photo shows a well-demarcated ovoid mass (arrow) with a mucous gelatinous texture with focal hemorrhage.

and some neutrophils (Fig. 2E). The mass had predominantly microcytic changes lined by attenuated flattened cells or no lining of cells and the cystic spaces were empty or filled with myxid materials (Fig. 2F, left). Size of the cystic spaces ranged from 300 $\mu \mathrm{m}$ to $1.2 \mathrm{~mm}$. Visceral pleura was free of tumor. The surrounding pulmonary parenchyma was normal. The fragmented partial thymectomy specimen showed a cystic structure, lined with cylindrical ciliated epithelial cells with surrounding normal-appearing thymic tissue with Hassall's corpuscles in the cyst wall. It was diagnosed as a unilocular thymic cyst.

Immunohistochemically, the spindle cells were positive for vimentin (prediluted, V9, Dako, Glostrup, Denmark) (Fig. 2F, right). They were all negative for matrix metalloproteinase (MMP)-2 (1:250, 4D3, Santa Cruz Biotechnology, Santa Cruz, CA, USA), MMP-9 (1:250, 2C3, Santa Cruz Biotechnology), pancytokeratin (prediluted, A1/A3, Dako), D2-40 (1:200, D240, Dako), TTF-1 (prediluted, 8G7G3, Dako), c-kit (1:40, T595, Novocastra, Newcastle upon Tyne, UK), S-100 protein (prediluted, polyclonal, Dako), desmin (prediluted, D33, Dako), smooth muscle actin (prediluted, 1A4, Dako), alpha-inhibin (1:50, BC/R1, Biocare Medical, Chicago, IL, USA), cal- retinin (prediluted, DAK-Calret1, Dako), CD34 (prediluted, QBEnd10, Dako), epithelial membrane antigen (prediluted, E29, Dako), CD68 (prediluted, PG-M1, Dako), CD99 (prediluted, 12E7, Dako), synaptophysin (prediluted, DAK-SYNAP, Dako), factor VIII-related antigen (prediluted, polyclonal, Dako), and factor XIII (1:50, E980.1, Novocastra). The myxoid stroma was stained with Alcian blue at $\mathrm{pH} 2.5$ with or without hyaluronidase, periodic-acid Schiff (PAS) and diastase-resistant PAS. The Ki-67 (prediluted, MIB-1, Dako) proliferation index was less than $0.1 \%$.

Ultrastructurally, the tumor was composed of several types of cells including spindle-shaped fibroblast-like cells (Fig. 3). The fibroblast-like spindle cells had a ruffled villus-like cell surface, and the cytoplasm was filled with rough endoplasmic reticulums, prominent Golgi complexes, and lysosomes. Amorphous flocculent and granular proteinaceous material and some collagen fibrils were observed in the extracellular spaces. Macrophages, mature lymphocytes, and some mast cells were also found. Cell junctions were not observed. Metastasis or embolic myxoma from the heart was excluded based on the absence of primary cardiac myxoma and the cytologic benignity of the pulmonary 

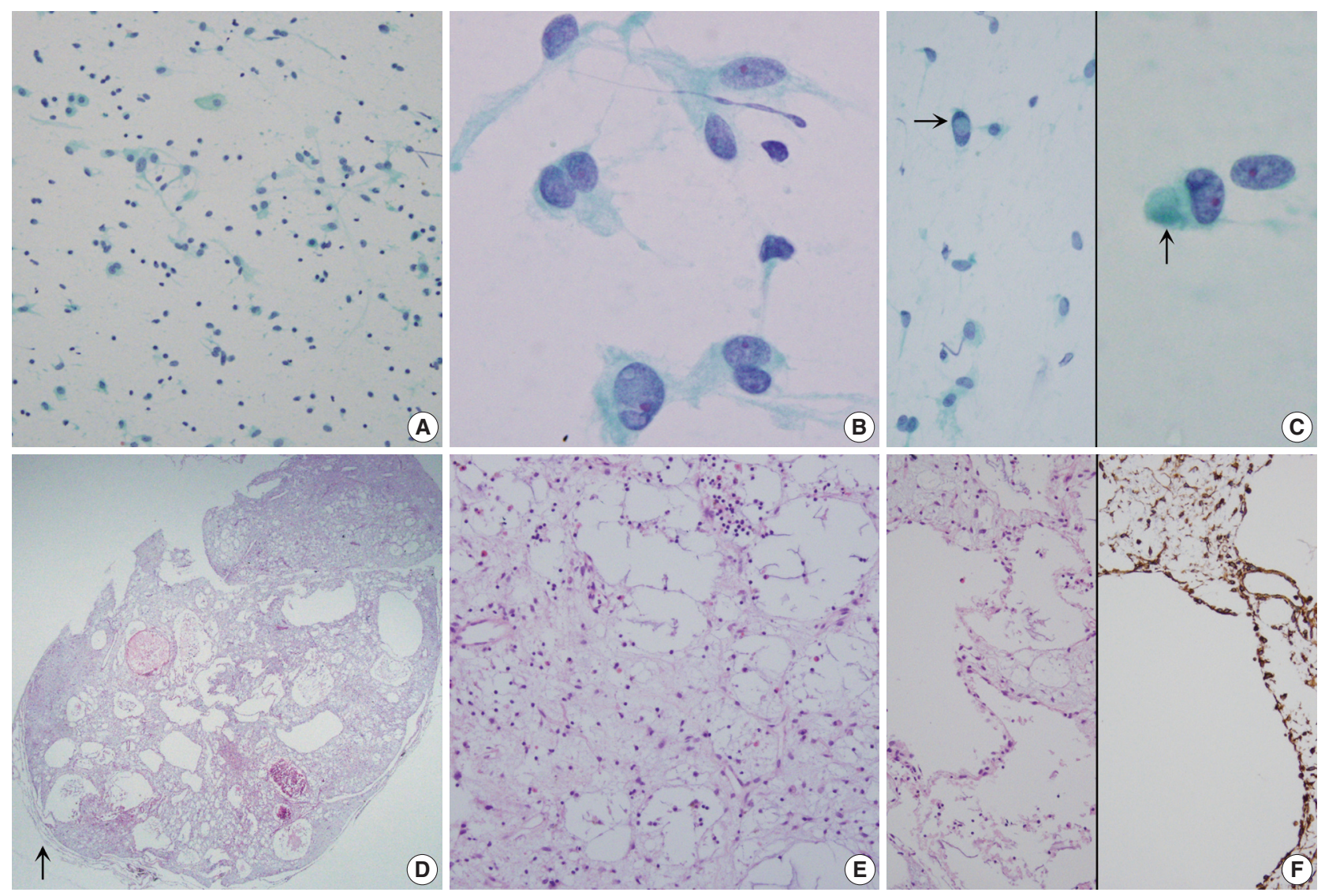

Fig. 2. (A-C) Touch imprint cytology. (A) Hypocellular smear shows many scattered inflammatory cells and macrophages. Note the background amorphous mucin-like metachromatic materials. (B) High-power view shows round to spindle cells having a moderate amount of granular cytoplasm and euchromatic round nuclei. (C) Note rare intranuclear inclusions (left, arrow) and cytoplasmic globular materials (right, arrow) (A-C, Papanicolaou stain). (D-F) Histologic findings. (D) Low-power view shows a well-demarcated bluish mass, and the mass is composed of predominantly chondromyxoid stroma. Arrow indicates normal pulmonary parenchyma. (E) High-power view shows that the mass is composed of abundant bluish chondromyxoid stroma and the paucicellular elements including scattered spindle cells, intermixed macrophages, mature lymphocytes, and some eosinophils. (F) Microcysts are surrounded by attenuated flattened cells or no lining (left, H\&E stain; right, Vimentin immunostain).

mass. Primary pulmonary myxoma was the diagnosis.

Chest CT taken 2 months after the operation revealed an interval increase in the extent of parenchymal consolidative lesion in the left upper lobe, which was accompanied by left pleural effusion. Cytology through pleurocentesis revealed metastatic adenocarcinoma. Brain magnetic resonance images revealed a small parenchymal mass with focal enhancement in the left cerebellar hemisphere. Chemotherapy with pemetrexed and cisplatin was performed, and radiation therapy was planned.

\section{DISCUSSION}

Touch imprints of the current case were paucicellular with abundant gelatinous mucin showing a folded pattern and scattered mucinophages. Simple capillaries were also aspirated. The differential diagnoses based on the cytologic features were mucin-rich lesions including mesenchymal tumors such as myxoma, hamartoma, extraskeletal myxoid chondrosarcoma (EMCS) and low cellular epithelial tumors such as mucinous epithelial tumor. ${ }^{1,6,7}$ Cytologic findings of pulmonary hamartoma are scantly cellular smears composed of bland-looking spindle and stellate cells and fibromyxoid materials in serosanguinous background. ${ }^{6}$ Pulmonary hamartoma is composed of predominantly chondroid connective tissue, adipocytes and fibroblasts with intervening nests of bland-looking epithelial cells. Cartilaginous component is rarely aspirated. Fine needle aspirates may show highly cellular smears showing epithelial cells with fine granular chromatin, mimicking a neuroendocrine neoplasm. Benign to low-grade mucinous epithelial tumors show a predominance of epithelial cells in the cytology, but mucinous cystic epithelial 


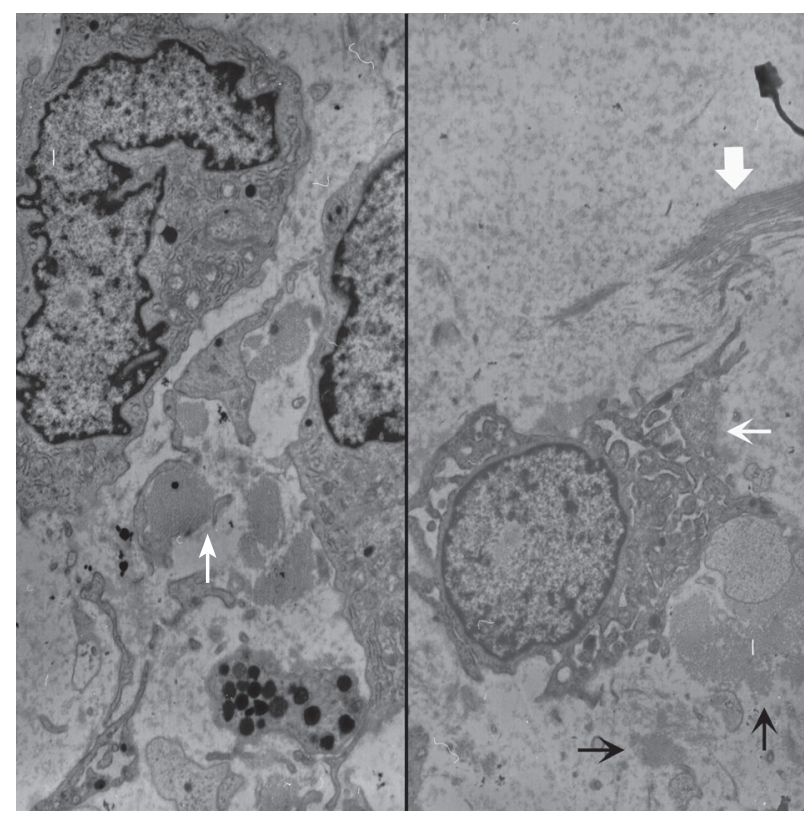

Fig. 3. Ultrastructural examination reveals spindle to round cells with round euchromatic nuclei and cytoplasm having many lysosomes, mitochondria, and rough endoplasmic reticulums. Extracellular amorphous mucin material (arrow) is observed (left, $\times 2,500$ ). Multiple ruffled cell surfaces (thin arrow) and abundant collagen fibrils (thick arrow) are also observed (right, $\times 3,500$ ).

tumors yield extremely low cellular mucin predominance with only a few floating epithelial cell nests. In such cases, diagnosis, particularly on scantly cellular cytology specimens, is exceedingly difficult. Mucin-rich smears must be searched carefully for clusters or single cells showing intracytoplasmic mucin vacuoles to ensure detection of mucinous cystic epithelial tumor. Cytologic findings of EMCS are composed of several fragments of polygonal to ovoid monotonous tumor cells with eccentrically located scalloped nuclei and peripheral fine cytoplasmic vacuoles embedded in a dense, metachromatic matrix as well as abundant myxoid materials. ${ }^{7}$ Vesicular nuclei or nuclear grooves or inclusions may occasionally be found in EMCS. ${ }^{8}$ Myxoid stroma can also be seen in myxoid liposarcoma. ${ }^{9}$ Atypical lipoblasts have central or eccentric nuclei or scalloped and vacuolated cytoplasm with high nucleus-cytoplasmic ratio, and can be seen clustered around branching, arborizing capillaries, unlike the simple capillaries seen in the current case.

The histologic differential diagnosis of this case was limited; pulmonary hamartoma and myxoma are the main differential diagnoses. Other differential diagnoses included EMCS, and low-grade myxofibrosarcoma. Pulmonary hamartoma is an important differential diagnosis. Like cardiac myxoma, pulmonary myxoma is composed of rich connective tissue derived from fi- broblasts and connective tissue mucin, whereas pulmonary hamartoma is composed of nonorganized epithelial cells and primitive mesenchymal spindle cells, including fibrous tissue, fat, smooth muscle, cartilage, and occasionally bone. Pulmonary myxoma consists of stellate or elongated fibroblast-like cells with abundant intercellular myxoid material. Variable amounts of inflammatory cell infiltration are a shared feature between myxoma and EMCS. EMCS is another important differential diagnosis, albeit extremely rare, in the lung. Pulmonary EMCS is a lowgrade tumor with multilobulated growth. EMCS is a low-cellularity tumor composed of uniform oval to spindle cells arranged in short anastomosing cords in an abundant myxoid matrix. No hyaline cartilaginous differentiation is seen. Mitoses are rarely found. Microscopic characteristics important for distinguishing between EMCS and myxoma include the different nature of myxoid stroma; extracellular mucin in myxoma is hyaluronidase-sensitive, which is stained with Alcian-blue, whereas chondromyxoid type of stroma, i.e., sulfated mucopolysaccharides in (Condroitin sulphate and keratan sulphate) EMCS is stained with Alcian-blue and is hyaluronidase-resistant.

To date, 10 cases of primary pulmonary myxoma, including the current case, have been reported. ${ }^{4,5}$ The ages of the patients ranged from 26 to 73 years (mean, 54.8 years). Five males and five females were reported. The tumor size ranged from 0.5 to $2.5 \mathrm{~cm}$ (mean, $1.59 \mathrm{~cm}$ ). All except for two endobronchial lesions occurred in pulmonary parenchyma; the present case presented with subpleural parenchymal mass. Half of the cases presented with nonspecific symptoms; hemoptysis, dyspnea, shortness of breath, chest pain or exacerbation of underlying chronic obstructive pulmonary disease. The remaining lesions were found incidentally. All but two with coexisting pulmonary adenocarcinomas showed excellent clinical outcome. Complete excision was curative and metastasis was not reported in all cases. The current case is similar to three cases reported by Shilo et al., which were reported as microcystic fibromyxoma, a histologic variant of pulmonary myxoma. Due to rarity and the subsequent lack of cumulative data on pulmonary microcystic myxoma, we suggest that cysts negative for both vascular markers and epithelial markers may be related to increased MMP activity in a microcystic variant of pulmonary myxoma, as in cardiac myxoma, showing excessive degradation of extracellular matrix and resulting in increased potential risk for metastatic embolism. ${ }^{10}$ Among 10 reported cases of pulmonary myxoma, metastasis had not yet been described. Accumulating prospective data on MMP-2 and MMP-9 immunoreactivities in pulmonary myxomas is necessary in order to evaluate the prognostic cor- 
relation.

While preoperative fine-needle aspiration is a well-established preoperative diagnostic technique in the epithelial neoplasm, it is limited in diagnosing soft tissue lesions due to scanty cellularity and the dense nature of the soft tissue lesion. Given the rarity of myxoma in comparison to common hamartoma, cytopathologists might ignore this entity in the differential diagnosis. Acknowledging the cytology of this benign lesion, pathologists should consider the possible shared features with other myxoid lesions, including malignant tumors.

\section{Conflicts of Interest}

No potential conflict of interest relevant to this article was reported.

\section{REFERENCES}

1. Meir K, Maly A, Doviner V, Maly B. Intraoperative cytologic diagnosis of unsuspected cardiac myxoma: a case report. Acta Cytol 2004; 48: 565-8.

2. Alaiti S, Nelson FP, Ryoo JW. Solitary cutaneous myxoma. J Am Acad Dermatol 2000; 43(2 Pt 2): 377-9.

3. Nadrous HF, Krowka MJ, Myers JL, Allen MS, Sabri AN. Tracheal myxoma: a rare benign tracheal tumor. Mayo Clin Proc 2004; 79: 931-3.
4. Özdoğan S, Fidan A, Saraç G, Çağlayan B, Uçmakli E. Concomitant occurrence of lung adenocarcinoma and endobronchial myxoma: a case report. Turk Respir J 2004; 5: 124-7.

5. Shilo K, Miettinen M, Travis WD, Timens W, Nogueira R, Franks TJ. Pulmonary microcystic fibromyxoma: report of 3 cases. Am J Surg Pathol 2006; 30: 1432-5.

6. Jin MS, Ha HJ, Baek HJ, Lee JC, Koh JS. Adenomyomatous hamartoma of lung mimicking benign mucinous tumor in fine needle aspiration biopsy: a case report. Acta Cytol 2008; 52: 357-60.

7. Handa U, Singhal N, Punia RS, Garg S, Mohan H. Cytologic features and differential diagnosis in a case of extraskeletal mesenchymal chondrosarcoma: a case report. Acta Cytol 2009; 53: 704-6.

8. Insabato L, Terracciano LM, Boscaino A, Mozzi RA, Angrisani P, Pettinato G. Extraskeletal myxoid chondrosarcoma with intranuclear vacuoles and microtubular aggregates in the rough endoplasmic reticulum: report of a case with fine needle aspiration and electron microscopy. Acta Cytol 1990; 34: 858-62.

9. Munjal K, Pancholi V, Rege J, Munjal S, Bhandari V, Nahar R. Fine needle aspiration cytology in mediastinal myxoid liposarcoma: a case report. Acta Cytol 2007; 51: 456-8.

10. Orlandi A, Ciucci A, Ferlosio A, Pellegrino A, Chiariello L, Spagnoli LG. Increased expression and activity of matrix metalloproteinases characterize embolic cardiac myxomas. Am J Pathol 2005; 166: 1619-28. 